\title{
Overall mechanical transfer function calculated for a spherical resonant gravitational waves antenna
}

\author{
José L. Melo a) \\ Instituto Nacional de Pesquisas Espaciais—INPE. São José dos Campos, SP, 12227-010, Brazil \\ Walter F. Velloso, Jr. ${ }^{\text {b) }}$ \\ Departamento de Física e Matemática-FFCLRP-USP, Ribeirão Preto, SP, 14040-901, Brazil \\ Odylio D. Aguiar ${ }^{\mathrm{C}}$ \\ Instituto Nacional de Pesquisas Espaciais—INPE. São José dos Campos, SP, 12227-010, Brazil
}

(Received 4 December 2003; accepted 30 March 2004; published online 24 May 2004)

\begin{abstract}
In this article, we describe the vibrational isolation system, the thermal link and the transducer mechanical structure, for the Schenberg resonant gravitational wave detector, which has been conceived modeled and analyzed using the finite element method. The dynamical equations corresponding to the structure have been solved using the MSC/NASTRAN software. In order to study the overall system behavior and the noise influence on it, we have carried out the analysis considering simultaneously all mechanical systems: vibrational isolation, thermal linking (between the dilution refrigerator and the suspension), and transducer-resonant mass mechanical coupling. Our calculation shows that a $300 \mathrm{~dB}$ attenuation level could be obtained. (C) 2004 American Institute of Physics. [DOI: 10.1063/1.1755444]
\end{abstract}

\section{INTRODUCTION}

The Graviton group is building the first Brazilian gravitational wave detector named Schenberg ${ }^{1}$ Antenna. This prototype detector will be composed by a suspended spherical metallic resonant mass with a $65 \mathrm{~cm}$ diameter weighting 1.15 ton. We expect to obtain a mechanical $Q$ about $20 \times 10^{6}$ with this resonant mechanical system. The detector has been designed to be sensitive in the range of frequencies 3100-3300 $\mathrm{Hz}$. Since a very large sensitivity level $\left(10^{-21} \mathrm{~m} / \mathrm{Hz}^{1 / 2}\right)$ must be achieved it was necessary to design a very efficient mechanical isolation system, capable of presenting a very large attenuation factor to the noise in the range of frequencies of interest.

The design was based on an idea that a multiple stage pendulum is capable of reducing, in the spectral region of detection interest, the mechanical vibrations that enter the system through the foundation point. Five cylinders joined together by $\mathrm{C}$ springs ${ }^{2}$ compose the system designed.

This system has some structural normal modes at low frequencies, which provide mechanical damping, ${ }^{3,4}$ but also presents a large number of resonance frequencies that correspond to the internal normal modes of each component of the structure (cylinders and $\mathrm{C}$ springs).

The finite element method (FEM) was used to model, design, and analyze all parts of the Schenberg antenna and to tune the frequencies of resonance far from the region of detection interest.

\footnotetext{
${ }^{a)}$ Electronic mail: ze@das.inpe.br

b)Electronic mail: velloso@dfm.ffclrp.usp.br

${ }^{c)}$ Electronic mail: odylio@das.inpe.br
}

\section{MODEL}

First we constructed a numerical finite element model for the simulation. ${ }^{5}$ After, using the results in an iterative way, we adapted the design in order to obtain the spectral characteristics required.

The basic constraints involved in the design ${ }^{6}$ were (1) to use cylinders small enough to positioning the first internal resonance at frequencies larger than and far from the detection frequency, and (2) to choose the geometrical parameters ${ }^{7}$ of the $\mathrm{C}$ springs (internal and external radius and height) and so adjust the elastic characteristics in a way to obtain a kind of spectral window, free from resonance frequencies, around the detection band.

Figure 1 shows our FE model for the Schenberg antenna and its isolation system suspending the $65 \mathrm{~cm}$ diameter sphere. The thermal link to the dilution refrigerator is also shown in this figure. This numerical model includes the resonant mass, the suspension vibration isolation system, the transducers, and the thermal link (which joins the dilution refrigerator to the suspension). The model was used for performing the static and the dynamical analysis in order to study the stress and the coupling between the internal mechanical parts of the detector and was also used to discover possible undesirable resonance frequencies in the detector sensitivity range. The model has about 10000 elements and 15000 nodes. One of the most interesting things in this modeling is that we have simulated both very large and very small pieces simultaneously in the same model such as the resonant mass $(1.15 \mathrm{~T})$ and the internal resonant transducer membrane (less than $1 \mathrm{~g}$ ). The hardest part during construction of the model was the transition meshing between large and small parts. Using this finite element model of the complete mechanical internal structure we calculated the stresses along the structure and the normal modes. 


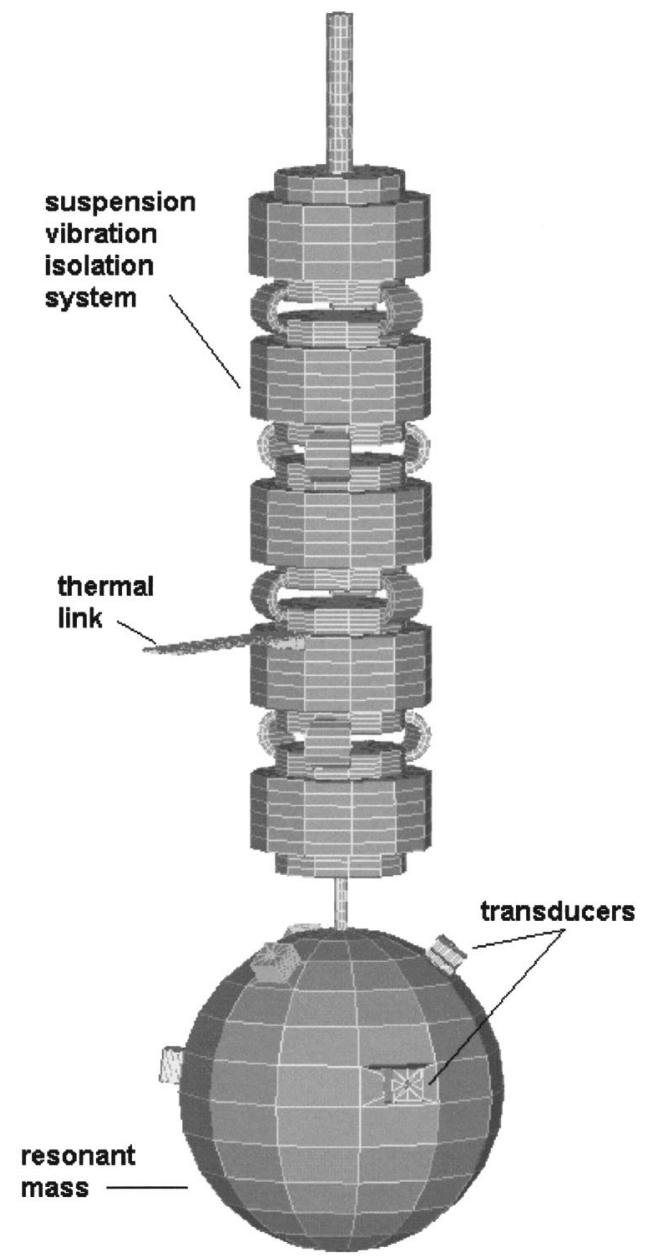

FIG. 1. The finite element model for the integrated mechanical systems. The sphere, the suspension isolation system, the thermal link, and the transducers have been represented in the same model.

After that, we calculated the frequency response to the whole system and used a simulated noise excitation signal, numerically applied to the top of the structure, in order to simulate the expected signal passing from the mechanical system foundation point, through the suspension system, until the transducer membrane.

\section{STATICS AND DYNAMICAL ANALYSIS}

The mechanical design of the structure was made with the purpose of obtaining a resonance free window in the detector sensitivity spectral range, where only the quadrupolar sphere frequencies can be present.
Since, to avoid second-order effects, the system has to be really far from the metal nonlinear elastic behavior region, we designed the pieces in order to have stresses along the structure always smaller than $20 \%$ of the typical yield stress for each metal or alloy used.

To achieve these two design targets both the static and dynamical analyses have been performed a few times and the structure changed in an iterative way until that the maximum admissible stress and the minimal required resonance free window was guaranteed.

The resonance free window around $3200 \mathrm{~Hz}$ (the detector characteristic frequency) is clearly noted in Fig. 2. In this range of frequencies, from 2625 to $3768 \mathrm{~Hz}$, only the first torsional $(2800-2900 \mathrm{~Hz})$ and quadrupolar $(3100-3200 \mathrm{~Hz})$ normal modes of the sphere are present. In a perfect sphere these modes are normally degenerated but our model, of course, includes the central hole which splits the frequencies.

\section{MODAL ANALYSYS}

Figures 3 and 4 show the modal shapes for two structural main modes calculated using the FEM. The mode shape shown in Fig. 3 is the pendular main resonance. The calculated frequency for this mode is about $1 \mathrm{~Hz}$. It is very low because this mode does not correspond to a free pendular mode. This pendular mode, in fact, acts as a spring-mass system and its characteristic frequency depends on the overall structural stiffness, which includes the suspension cable and the cylinder and $\mathrm{C}$-springs modules, as well as on the inverse of the cylinders and sphere masses. Since the sphere mass is very high (about $1150 \mathrm{~kg}$ ) and the overall structure is relatively soft to movements in the horizontal plane, the frequency of the main pendular mode is very small. This low frequency pendular mode is an important characteristic of the suspension system, since it corresponds to a very-low pass filter and results, at the $3000-3300 \mathrm{~Hz}$ range, in a large isolation factor to any kind of noise that could produce horizontal movements at the antenna foundation points. The first torsional main mode, at $14 \mathrm{~Hz}$, which is not shown here, performs an analogous function, efficiently filtering the high frequency noises which could shake the antenna foundation points and produce torsional movements on the suspension structure and on the sphere. These two very low frequency modes (pendular and torsional) carry out the main vibration isolation in the horizontal plane ( $X$ and $Y$ axes).

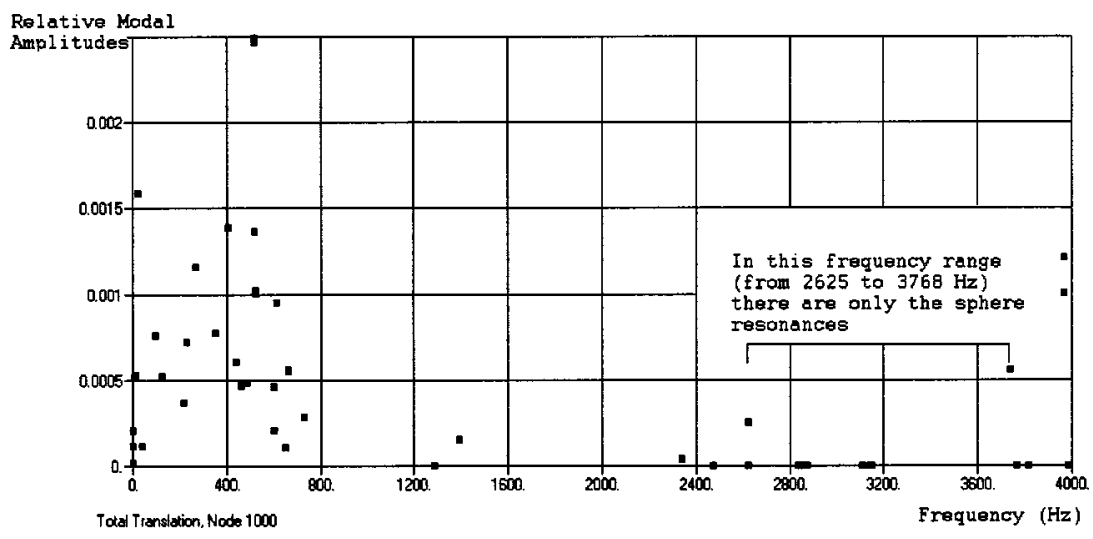

FIG. 2. Integrated system normal modes between 0 and $3400 \mathrm{~Hz}$ obtained from FEM analysis. The points correspond to the eigenvalues (mode frequencies) plotted vs the normalized relative amplitudes of the modes. 

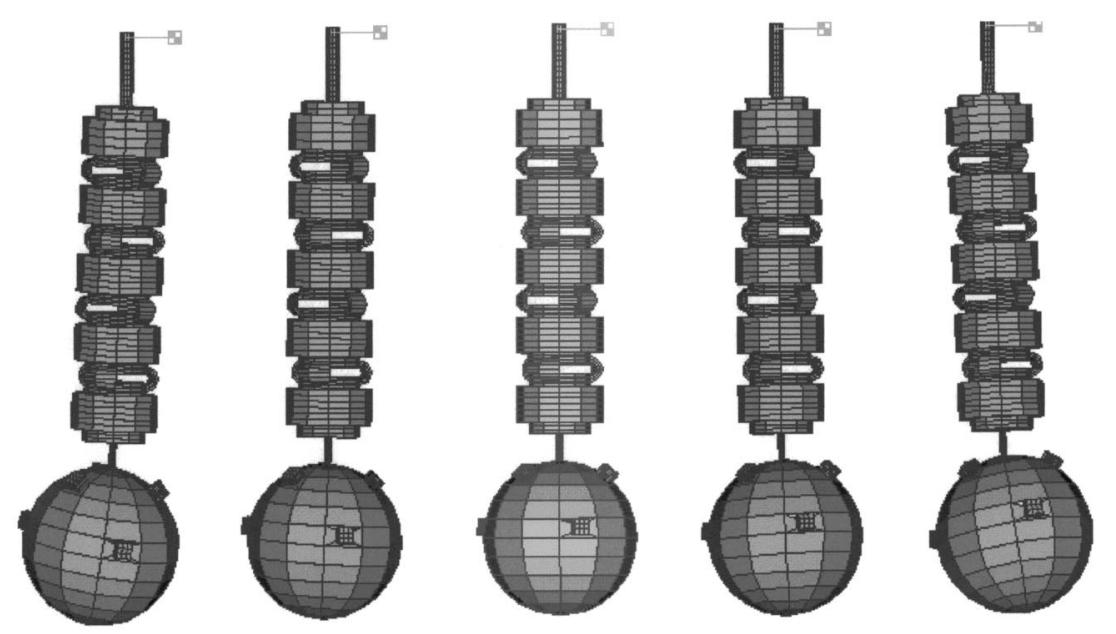

FIG. 3. The lower pendular structural normal mode at $1 \mathrm{~Hz}$. The amplitudes have been arbitrarily enhanced to emphasize the modal shape.
The mode shown in Fig. 4 is the longitudinal main mode. Also in this case, the movement corresponds to a spring-mass system whose characteristic frequency is determined by the stiffness of the $\mathrm{C}$-springs and by the masses of the sphere, cylinders and $\mathrm{C}$-springs. Again, once the masses involved are so high and since the C-springs have been designed to be as soft as possible, the calculated resonant frequency is low $(36.5 \mathrm{~Hz})$. These very-low pass filters carry out the main vibration isolation in the vertical direction $(Z$ axis).

The high efficiency of isolation represented by these low frequency modes and also by the corresponding high order modes could be harmed by the internal resonances of the cylinders and C-springs themselves. These movements could excite the suspension cable's internal resonances so transferring noise to the sphere. However, the contact between the suspension cable and the sphere will always play the role of a node to the cable's internal resonant modes. This means that the cable's internal mode amplitudes at this point will be almost zero, and since the cable is to be attached as close as possible to the sphere barycenter, the noise transfer by this process will be minimized. In fact, the isolation performance could be spoiled by an estimated factor ${ }^{8} f=(\delta / R)$ where $\delta$ is the distance from the center of mass and $R$ is the sphere's radius. So, $\delta \cong 0$ means the best isolation. If the sphere $(R=325 \mathrm{~mm})$ is suspended, for example, $1 \mathrm{~mm}$ off the barycenter, the factor $f$ would be about $1 / 325 \cong 0.003$.

\section{FREQUENCY RESPONSE TO CRITICAL NOISES AND THE TRANSFER FUNCTION}

There are two critical points for the mechanical vibration noise to enter the system: through the vibration isolation system itself or through the thermal link. In the first case, the main noise to be considered is the seismic one. In the second case, the seismic noise is present, but we must consider also the low frequency $(<10 \mathrm{~Hz})$ dilution refrigerator internal noise. Seismic noise signal amplitude, of course, depends on the geographic location and is known to have a $\nu^{-2}$ dependence ${ }^{9}$ law.

The dilution refrigerator noise however is not well known, since it depends on the details of the refrigerator construction and internal working.

Then, the next step in our simulation procedure was the assumption of a relatively strong white signal between 0 and $10 \mathrm{~Hz}$ (see Fig. 5) with the purpose of simulating the internal refrigerator noise. This white signal, superposed to the $\nu^{-2}$ dependence of seismic noise, corresponds to an overestimation and it was numerically applied to the model in order to estimate the frequency response of the thermal link.

As usually done in FEM analyses, a structural damping factor of 5\% of the critical damping has been assumed just to prevent infinite amplitudes. The frequency response was calculated considering also the presence of the parametric transducers, ${ }^{10}$ attached to the resonant mass of Schenberg

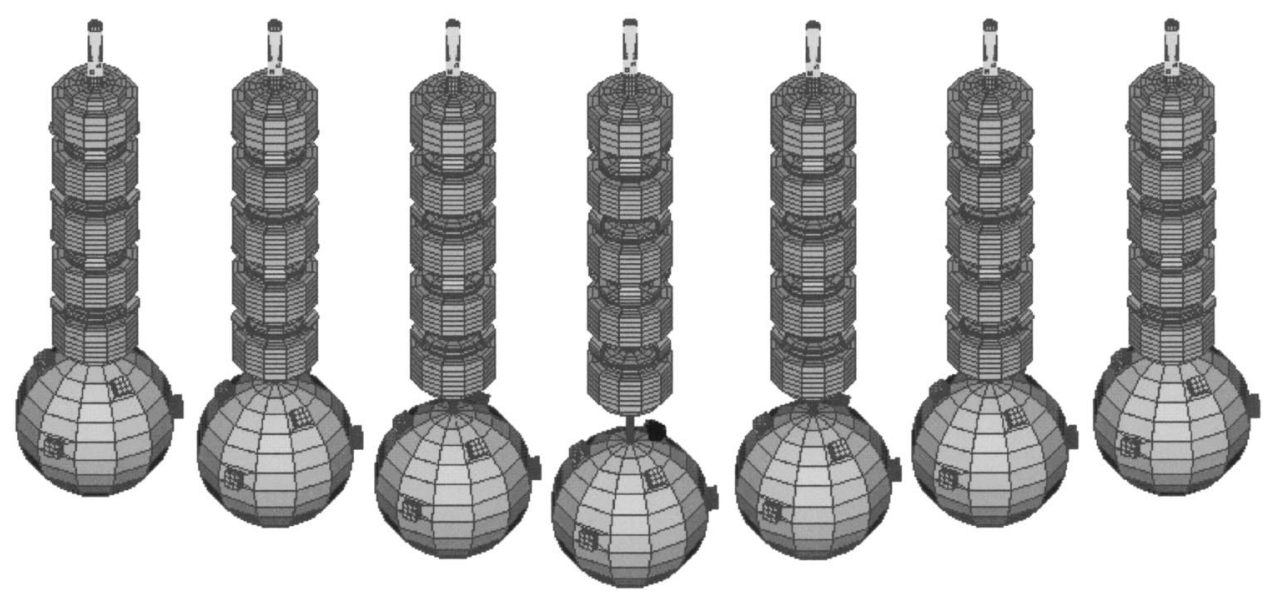

FIG. 4. The lower longitudinal structural normal mode at $36 \mathrm{~Hz}$. The amplitudes have been arbitrarily enhanced to emphasize the modal shape. 


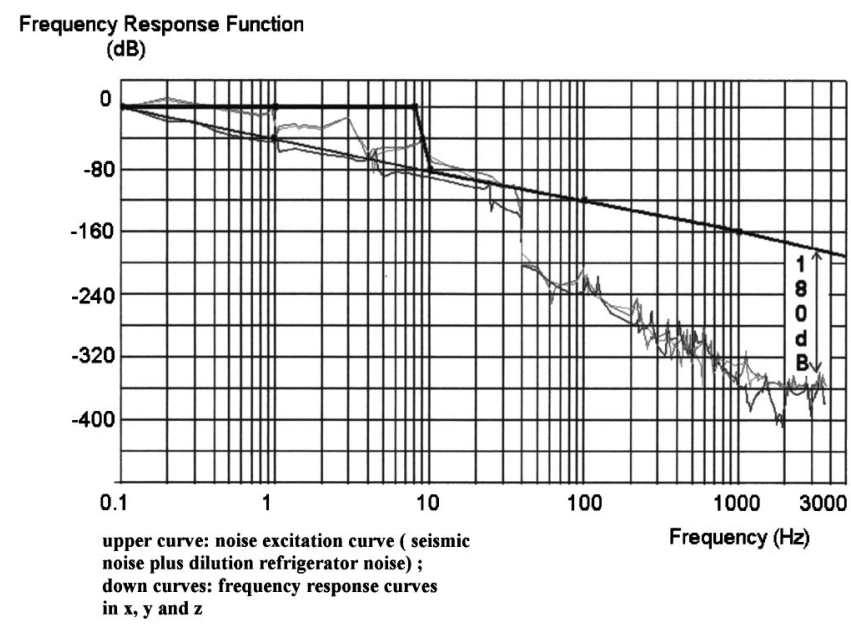

FIG. 5. Calculated frequency response curves. An overestimated noise, introduced in the structure by the thermal link connected to the suspension, has been considered.

detector. Also a microwave cavity was modeled on the transducer mechanical structure which is composed of two high mechanical $Q$ resonators. The main mode frequencies of both those resonators must be adjusted to the value corresponding to the mean of the five sphere quadrupolar frequencies, in order to optimize ${ }^{11}$ the energy transfer from the sphere to the transducers if a hypothetical gravitational wave excite the sphere quadrupolar modes.

Each microwave cavity has a very thin, and light, front wall that corresponds to the transducer's second resonator. When excited by the sphere resonance, this kind of membrane will vibrate and its vibration will modulate a microwave carrier signal injected into the cavity.

In Fig. 6 the results of the final simulation are plotted. The six curves correspond to the $x, y, z$, and $\theta_{x}, \theta_{y}, \theta_{z}$ transfer functions for the entire system, calculated comparing the frequency responses in an element next to the excitation point and in an element on transducer's membrane. In all the cases the attenuation levels reached $300 \mathrm{~dB}$.

The low frequency main resonances cited in session IV are visible and it is easy to see the high filtering effect they produce. In spite of the large isolation factor, it is also possible to see some of the large frequency internal resonances, since, to avoid numerical precision limitations inherent in the computational method, we have used the high excitation noise signal cited. However, it is clear that these internal resonances do not represent any harm to the isolation factor from 3000 to $3300 \mathrm{~Hz}$ where the frequencies of detection interest are.

\section{DISCUSSION}

Using the FEM has been a valuable designing tool to implement the vibration isolation system for the Schenberg detector.

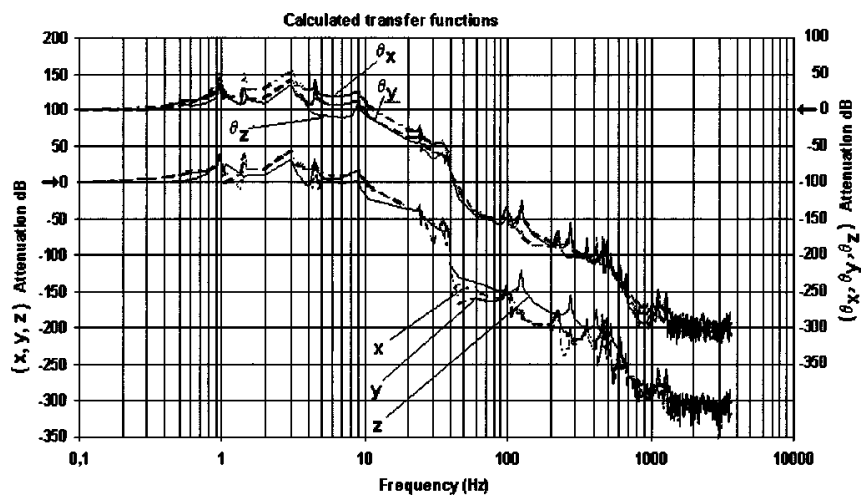

FIG. 6. Calculated overall $\left(x, y, z\right.$, plus $\left.\theta_{x}, \theta_{y}, \theta_{z}\right)$ transfer functions for the integrated system. In order to obtain more clarity, the scale origin for $\theta_{x}$, $\theta_{y}$, and $\theta_{z}$ has been moved up $100 \mathrm{~dB}$.

We were able to evaluate the mechanical attenuation, to calculate the transfer function for the system and to estimate the noise level that will pass through the structure.

Since the seismic noise level ${ }^{12}$ at the detection frequency is expected to be about $10^{-14} \mathrm{~m} \mathrm{~Hz}^{-1 / 2}$, the $300 \mathrm{~dB}$ attenuation level at $3200 \mathrm{~Hz}$ guarantees that the mechanical noise of seismic or cultural origins will not be restrictive to detect gravitational waves at $10^{-22} \mathrm{~m} \mathrm{~Hz}^{-1 / 2}$ level. In other words, this means that the detector noise will be dominated by the thermal noise.

The vibration isolation system we have designed is being constructed and the Graviton Group is setting up the Schenberg detector.

\section{ACKNOWLEDGMENT}

The authors would like to thank FAPESP (Fundação de Amparo à Pesquisa do Estado de São Paulo) under Grant Nos. 98/13468-9 and 01/12606-3, for supporting this work.

${ }^{1}$ O. D. Aguiar et al., Class. Quantum Grav. 19, 1949 (2002).

${ }^{2}$ V. C. Viscont, Ph.D. thesis, University of Padue, Italy, 1999

${ }^{3}$ C. A. Cantley, J. Hough, N. A. Robertson, and A. Greenhalgh, Rev. Sci. Instrum. 63, 2210 (1992).

${ }^{4}$ T. L. Aldcroft, P. F. Michelson, R. C. Taber, and F. A. McLoughlin, Rev. Sci. Instrum. 63, 8 (1992)

${ }^{5}$ J. L. Melo, Ph.D. thesis, Instituto Nacional de Pesquisas Espaciais, Brazil, 2002

${ }^{6}$ W. F. Velloso, Jr., J. L. Melo, and O. D. Aguiar, Rev. Sci. Instrum. 71, $2552(2000)$

${ }^{7}$ J. L. Melo, W. F. Velloso, Jr., and O. D. Aguiar, Class. Quantum Grav. 19, 1985 (2002)

${ }^{8}$ E. Coccia, Rev. Sci. Instrum. 55, 1980 (1984).

${ }^{9}$ A. Araya, K. Kawabe, T. Sato, N. Mio, and K. Tsubono, Rev. Sci. Instrum. 64, 1337 (1993).

${ }^{10}$ J. P. Richard, Phys. Rev. Lett. 52, 165 (1984).

${ }^{11}$ J. L. Melo, W. F. Velloso, Jr., O. D. Aguiar, and A. U. Lucena, Class. Quantum Grav. (to be published).

${ }^{12}$ D. G. Blair, D. E. McClelland, H. A. Bachor, and R. J. Sandeman, in The Detection of Gravitational Waves, edited by D. G. Blair (Cambridge University, Cambridge, England, 1991), p. 43. 